\title{
Co-Channel Interference Suppression for Coded OFDM Systems over Frequency-Selective Slowly Fading Channels
}

\author{
Ching-Sheng $\mathrm{Ni}^{1}$ and Kwang-Cheng Chen ${ }^{2}$ \\ ${ }^{1}$ csni@santos.ee.ntu.edu.tw2 ${ }^{2}$ chenkc@cc.ee.ntu.edu.tw \\ Institute of Communications Engineering, National Taiwan University, Taiwan, R.O.C.
}

\begin{abstract}
Inspired by multiuser detection theory, we propose the linear minimum mean-square error (LMMSE) multiuser detector (MUD) to suppress co-channel interference (CCI) with unknown signal format for coded OFDM systems over single input/single output frequency-selective slowly fading channels by considering each sub-carrier as an individual user. To eliminate the residual interference contributed by other sub-carriers at the output of the LMMSE MUD, we propose the multistage interference cancellation (MIC) algorithm which makes use of the reliable tentative decisions of other sub-carriers generated from the decoder outputs of the first LMMSE MUD stage. To further improve the performance, we propose the enhanced multistage interference cancellation (EMIC) algorithm which is the LMMSE estimate of the information bit provided that the signal components of other sub-carriers are precisely removed from the received signal. From computer simulations, the MIC and the EMIC algorithms effectively improve the performance of the LMMSE MUD. Furthermore, the performance of the EMIC is very close to the optimal when the desired OFDM signal employs Quadrature phase-shift keying (QPSK) or 16-ary quadrature amplitude modulation (16-QAM).
\end{abstract}

\section{INTRODUCTION}

Orthogonal frequency division multiplexing (OFDM) is a promising technology to next generation wireless communications and had been extensively applied in a lot of wireless communications systems such as IEEE $802.11 \mathrm{a} / \mathrm{g}$ in wireless local area networks (WLAN) and HIPERLAN/2 in broadband radio access networks. In OFDM systems, information bits are split into a bank of $N_{c}$ mutually orthogonal sub-carriers and transmitted simultaneously. With the introduction of cyclic prefix (CP) between symbols, the inter-symbol interference (ISI) and inter-carrier interference (ICI) can be easily avoided, which saves the complexity of receivers. In addition, inheriting the property of high spectral efficiency from multi-carrier systems, OFDM systems provide high data rate transmission which further suggests the practical applications to future advanced communications systems.

However, due to the extensive applications to the frequency bands with other coexistent wireless communications systems, the wireless OFDM systems unavoidably face the challenges of the external co-channel interference (CCI) when overlay networks are deployed. For example, considering IEEE $802.11 \mathrm{~g}$ operating at $2.4 \mathrm{GHz}$ industrial, science, medical

This research is supported by National Science Council under the contract NSC 91-2213-E-002-102
(ISM) band, the communications devices of IEEE 802.11b and IEEE 802.15 would introduce interference to severely degrade the performance of IEEE 802.11g. Therefore, suppressing CCI has become a critical issue to preserve the advantages of wireless OFDM systems.

In suppressing CCI for OFDM systems, antenna arrays were proposed in [1], [2], where the outputs at the demodulators of different received antennas of a certain sub-carrier are linearly combined based on minimum mean-square error (MMSE) criterion as an estimate of its information bit without any knowledge of CCI. Beamforming techniques were proposed in [2], [3] to blind out CCI from certain directions by making use of spatial signatures of desired user and CCI. Given the knowledge of signal formats and channels of CCI, percarrier maximum likelihood estimation (pc-MLE) [4], [5], percarrier successive interference cancellation (pc-SIC) [5] and per-carrier parallel interference cancellation (pc-PIC) [6] were proposed for OFDM systems with space division multiple access (SDMA). However, when the number of the interfering signals with unknown signal formats on each sub-carrier are larger or equal to the number of the received antennas, the algorithms in [1], [2], [3] become not effective and the algorithms in [4], [5], [6] is not applicable.

On the other hand, the linear minimum mean-square error (LMMSE) multiuser detector (MUD) was shown to be nearfar resistant over single input/single output channels provided that the received waveform of the desired user is not spanned by the received waveforms of other users [7]. And more importantly it can be implemented without side information of CCI through estimating the autocorrelation matrix of the received signal or through data-aided (DA), decision-driven (DD) and blind adaptive algorithms as shown in [7], [8]. In [9], [10], [11], the LMMSE MUD were proposed to effectively suppress both narrow-band and wide-band CCI in DS/CDMA systems. In this paper, motivated by these observations, we propose algorithms based on the LMMSE MUD to suppress CCI with unknown signal format for wireless coded OFDM systems by considering each sub-carrier as an individual user. Although to jointly consider multiuser detection and antenna arrays processing is the optimal way, in order to assess the capability of MUDs in CCI suppression for OFDM systems, we consider a single input/single output channel throughout this paper. The LMMSE MUD are proposed to suppress CCI in 
this scenario. Although the signal format of CCI is unknown, we can further eliminate residual interference contributed by other sub-carriers by making use of tentative decisions, which is inspired by the nonlinear DD MUDs. Based on this idea, we propose the multistage interference cancellation (MIC) and the enhanced multistage interference cancellation (EMIC) algorithms with the LMMSE MUD as their first stage to further improve the performance.

The rest of the paper is organized as follows. In Section II, the signal models and assumptions are introduced. In Section III, the LMMSE MUD, the MIC and the EMIC algorithms are developed. In Section IV, numerical results are presented to assess the performance of the proposed algorithms for OFDM systems with forward error correction (FEC) code. In Section $\mathrm{V}$, we make some conclusions.

\section{Assumptions And Signal Models}

\section{A. Assumptions}

We consider a scenario where one OFDM desired signal with a single input/single output channel and one cyclostationary interference with period $M T_{0}$ share a common frequency band, where $M$ is a positive integer known to the receiver and $T_{0}$ is the OFDM symbol duration. The receiver is assumed to precisely acquire synchronization information of the desired signal including symbol timing, carrier frequency, carrier phase and channel impulse response, which implies the received waveforms of OFDM sub-carriers are precisely known to the receiver. The channel impulse response of the desired signal is assumed frequency-selective fading and timeinvariant in our observation interval of interest. The maximum delay spread of the channel is assumed smaller than the length of CP such that ISI and ICI can be avoided by removing CP.

\section{B. Signal models}

The precisely synchronized low-pass equivalent received signal is given as

$$
y(t)=s(t)+i(t)+n(t)
$$

where $n(t)$ is the complex-valued additive white Gaussian noise with independent real part and imaginary part and each part has power spectral density $N_{0} / 2, i(t)$ is the cyclostationary CCI with period $M T_{0}$ and $s(t)$ is the desired OFDM signal given by

$$
s(t)=\sum_{p=0}^{P-1} \sum_{q=0}^{N_{c}-1} a(p, q) \sum_{k=0}^{K-1} c_{k} h_{q}\left(t-p T_{0}-k / W\right)
$$

where $h_{q}(t)$ is the transmitted waveform of the $q$ th sub-carrier given by

$$
\begin{array}{r}
h_{q}(t)=\left\{\begin{array}{cc}
\frac{1}{\sqrt{T_{0}}} e^{j 2 \pi q t / T_{1}} & 0 \leq t<T_{0} \\
0 & \text { else }
\end{array}\right. \\
q=0,1, \ldots, N_{c}-1,
\end{array}
$$

$P$ is the number of symbols of the OFDM signal, $N_{c}$ is the number of sub-carriers of an OFDM symbol, $a(p, q) \in\{ \pm 1\}$ is the uncorrelated coded information bit of the $q$ th sub-carrier of the $p$ th OFDM symbol, $1 / T_{1}$ is the frequency separation of successive OFDM sub-carriers, $T_{0}-T_{1}$ is the length of $\mathrm{CP}, c_{0}, c_{1}, \ldots, c_{K-1}$ are mutually independent complex-valued Gaussian random variables corresponding to fading channel coefficients of different delay paths and $K$ is the number of resolvable paths of OFDM signal and is approximately equal to $\left\lfloor T_{d} W\right\rfloor+1$ where $T_{d}$ and $W$ are respectively the maximum delay spread of the channel and the bandwidth of the OFDM signal and $\lfloor x\rfloor$ is defined as the largest integer smaller than $x$ [12]. In this paper, since we primarily focus on suppressing unwanted external CCI for OFDM systems, these assumptions on signal models will ease the development of the receiver. These assumptions are also reasonable for high data rate OFDM applications with small mobility such as $802.11 \mathrm{~g}$ WLAN and HIPERLAN/2.

\section{INTERFERENCE SUPPRESSION ALGORITHMS}

In this section, the LMMSE MUD of the desired sub-carrier based on the sliding window observation [13] of period $M T_{0}$ is proposed. To improve the performance of the LMMSE MUD, the MIC and the EMIC with the LMMSE MUD as their first stage are developed.

\section{A. The LMMSE MUD}

After removing $\mathrm{CP}$, the vectored representation of received signal is generated by taking discrete time samples in $\{t$ : $\left.t \in \bigcup_{p=0}^{P-1}\left[(p+1) T_{0}-T_{1},(p+1) T_{0}\right]\right\}$. The $L \times 1$ received vector $\mathbf{y}(p)$ of the $p$ th OFDM symbol duration is defined as $\mathbf{y}(p) \equiv\left[y\left((p+1) T_{0}-T_{1}+0 T_{0} / L\right) y\left((p+1) T_{0}-\right.\right.$ $\left.\left.T_{1}+1 T_{0} / L\right) \ldots y\left((p+1) T_{0}-T_{1}+(L-1) T_{0} / L\right)\right]^{T}, p=$ $0,1, \ldots, P-1$. Since the received signal is cyclostationary with period $M T_{0}$. To make the coefficients of LMMSE MUD independent of time, the period of sliding windows must be an integer multiple of $M T_{0}$. We let the period of sliding window be $M T_{0}$ without loss of generality.

The LMMSE MUD of the information bit $a\left(p^{\prime}+i M, q\right)$, $p^{\prime}=0,1, \ldots, M-1, q=0,1, \ldots, N_{c}-1$, based on the $i$ th $L M \times$ 1 sliding window observation $\mathbf{y}_{w}(i) \equiv\left[\mathbf{y}^{T}(i M) \mathbf{y}^{T}(i M+\right.$ 1) $\left.\ldots \mathbf{y}^{T}(i M+M-1)\right]^{T}$, has the form

$$
\widetilde{a}_{L M M S E}\left(p^{\prime}+i M, q\right)=\mathbf{v}_{L M M S E}^{T}\left(p^{\prime}+i M, q\right) \mathbf{y}_{w}(i)
$$

where $\mathbf{v}_{L M M S E}\left(p^{\prime}+i M, q\right)$ is chosen to minimized the meansquare error (MSE) given by

$$
\begin{aligned}
& \mathbf{v}_{L M M S E}\left(p^{\prime}+i M, q\right) \\
& \quad=\arg \min _{\mathbf{v} \in C^{L M \times 1}} E\left[\left|a\left(p^{\prime}+i M, q\right)-\mathbf{v}^{T} \mathbf{y}_{w}(i)\right|^{2}\right] .
\end{aligned}
$$

By taking complex gradient of the MSE with respect to the dummy variable $\mathbf{v}$ and applying the wide-sense stationary property of $\mathbf{y}_{w}(i)$ with respect to $i, \mathbf{v}_{L M M S E}^{T}\left(p^{\prime}+i M, q\right)$ is shown to be

$$
\begin{aligned}
& \mathbf{v}_{L M M S E}^{T}\left(p^{\prime}+i M, q\right) \\
& \quad=E\left[\left(\mathbf{y}_{w}^{H}(i) a\left(p^{\prime}+i M, q\right)\right] E^{-1}\left[\mathbf{y}_{w}(i) \mathbf{y}_{w}^{H}(i)\right]\right. \\
& \quad=\mathbf{s}_{w}^{H}\left(p^{\prime}, q\right) E^{-1}\left[\mathbf{y}_{w}(0) \mathbf{y}_{w}^{H}(0)\right] \\
& \quad=\mathbf{s}_{w}^{H}\left(p^{\prime}, q\right) \mathbf{R}^{-1}
\end{aligned}
$$


where (.) ${ }^{H}$ denotes the conjugate-transpose operator and $\mathbf{s}_{w}\left(p^{\prime}, q\right)$ is the $L M \times 1$ vectored representation of received waveform of the information bit $a\left(p^{\prime}+i M, q\right)$ in the $i$ th sliding window given as

$$
\mathbf{s}_{w}\left(p^{\prime}, q\right)=\left[\begin{array}{lllll}
\overbrace{}^{\underline{\mathbf{0}}} \ldots \underline{\mathbf{0}} & \mathbf{s}_{0, q}^{T} \overbrace{\underline{\mathbf{0}} \ldots}^{M-\underline{\mathbf{0}}^{\prime}-1}
\end{array}\right]^{T}
$$

where $\underline{\mathbf{0}}$ is a $1 \times L$ zero vector and $\mathbf{s}_{0, q}$ is the $L \times 1$ received vector of the information bit $a(0, q)$. The coefficients of the LMMSE MUD can be obtained according to (6) by directly inverting the estimate of autocorrelation matrix $\mathbf{R}$. To save the implementation complexity of matrix inversion, adaptive algorithms are widely applied, including data-aided (DA), decision-driven (DD) [7], [14] and blind [8], [10], [11], [15], [16] adaptive algorithms.

In addition to minimize the MSE, the LMMSE MUD was shown to maximize the output signal-to-interference plus noise ratio (SINR) among all linear filters [13]. The output SINR of the LMMSE MUD of the information bit $a\left(p^{\prime}+i M, q\right)$, $p^{\prime}=0,1, \ldots, M-1, q=0,1, \ldots, N_{c}-1$, is shown to be

$$
\begin{aligned}
S I N & R_{L M M S E}\left(p^{\prime}+i M, q\right) \\
& =\frac{\left[\mathbf{s}_{w}^{H}\left(p^{\prime}, q\right) \mathbf{R}^{-1} \mathbf{s}_{w}\left(p^{\prime}, q\right)\right]^{2}}{\mathbf{s}_{w}^{H}\left(p^{\prime}, q\right) \mathbf{R}^{-1} \mathbf{s}_{w}\left(p^{\prime}, q\right)-\left[\mathbf{s}_{w}^{H}\left(p^{\prime}, q\right) \mathbf{R}^{-1} \mathbf{s}_{w}\left(p^{\prime}, q\right)\right]^{2}} .
\end{aligned}
$$

When $\widetilde{a}_{L M M S E}\left(p^{\prime}+i M, q\right)$ serves as a soft input to the decoder, the SINR in (8) should be invoked to generate soft-input information for the decoder. Alternatively, $\widetilde{a}_{L M M S E}\left(p^{\prime}+\right.$ $i M, q)$ may pass through a threshold detector to generate a hard input to the decoder.

\section{B. The MIC Algorithm}

Although the LMMSE MUD applies optimal linear filtering, certain sub-carriers with low received amplitude may be completely buried in multiple access interference (MAI). This motivates us to develop nonlinear algorithms to improve the performance. Several types of nonlinear decision-driven MUDs were proposed to enhance the performance of linear MUDs by making use of tentative or final decisions, including the successive interference cancellation (SIC) MUDs and the multistage MUDs [7]. In our scenario, however, since each sub-carrier is subjected to a frequency-flat channel, both the SIC MUDs and the multistage MUDs suffer from serious erroneous cancellation if the tentative decisions are generated without incorporating FEC. Through incorporating FEC, by obtaining frequency diversity gain over sub-channels, the tentative decisions provide much higher reliability. Therefore, we propose the MIC algorithm incorporating FEC based on the multistage MUDs.

The idea of the MIC is to cancel the residual interference contributed by other sub-carriers at the output of the LMMSE MUD by making use of the signal formats and reliable tentative decisions of other sub-carriers from the first stage. In the presence of the external interfering signals with unknown signal formats, the MIC employs the LMMSE MUD as a reliable first stage. The estimate of the information bit $a\left(p^{\prime}+i M, q\right), p^{\prime}=0,1, \ldots, M-1, q=0,1, \ldots, N_{c}-1$, at the output of the second stage of the MIC has the form

$$
\begin{aligned}
\widetilde{a}_{M I C}\left(p^{\prime}+i M, q\right) & \\
= & \mathbf{v}_{L M M S E}^{T}\left(p^{\prime}+i M, q\right) \\
& {\left[\mathbf{y}_{w}(i)-\sum_{u=0, u \neq p^{\prime}}^{M-1} \sum_{v=0, v \neq q}^{N_{c}-1} \hat{a}(u+i M, v) \mathbf{s}_{w}(u, v)\right] }
\end{aligned}
$$

where $\hat{a}(u+i M, v)$ is the tentative decision of $a(u+i M, v)$, generated from the decoder outputs of the first LMMSE MUD stage. The primary advantage of the MIC is that it shares the same coefficients of the LMMSE MUD such that its increment of the implementation complexity over the LMMSE MUD is only contributed by the generation of the tentative decisions involving FEC decoding.

Let us assume perfect tentative decisions and denote $G\left(p^{\prime}, q\right) \equiv \sum_{u=0}^{M-1} \sum_{v=0}^{N_{c}-1}\left[\mathbf{s}_{w}^{H}\left(p^{\prime}, q\right) \mathbf{R}^{-1} \mathbf{s}_{w}(u, v)\right]^{2}$. The output SINR of the MIC of the information bit $a\left(p^{\prime}+i M, q\right)$, $p^{\prime}=0,1, \ldots, M-1, q=0,1, \ldots, N_{c}-1$, is shown to be

$$
S I N R_{M I C}\left(p^{\prime}+i M, q\right)=\frac{\left[\mathbf{s}_{w}^{H}\left(p^{\prime}, q\right) \mathbf{R}^{-1} \mathbf{s}_{w}\left(p^{\prime}, q\right)\right]^{2}}{\mathbf{s}_{w}^{H}\left(p^{\prime}, q\right) \mathbf{R}^{-1} \mathbf{s}_{w}\left(p^{\prime}, q\right)-G\left(p^{\prime}, q\right)} .
$$

The output SINR improvement of the MIC over the LMMSE MUD is observed from the denominators in (8) and (10). The MIC eliminates the residual interference contributed by other sub-carriers at the output of the LMMSE MUD such that the residual interference power is reduced from $\mathbf{s}_{w}^{H}\left(p^{\prime}, q\right) \mathbf{R}^{-1} \mathbf{s}_{w}\left(p^{\prime}, q\right)-\left[\mathbf{s}_{w}^{H}\left(p^{\prime}, q\right) \mathbf{R}^{-1} \mathbf{s}_{w}\left(p^{\prime}, q\right)\right]^{2}$ to $\mathbf{s}_{w}^{H}\left(p^{\prime}, q\right) \mathbf{R}^{-1} \mathbf{s}_{w}\left(p^{\prime}, q\right)-G\left(p^{\prime}, q\right)$ while the signal power is kept unchanged. The average bit error rate (BER) performance improvement of the MIC over the LMMSE MUD through computer simulations will also be shown in Section IV.

\section{The EMIC Algorithm}

When the tentative decisions are highly reliable at high $E_{b} / N_{0}$, which thanks to the effectiveness of the LMMSE MUD, we can assume that the signal components of other sub-carriers can be precisely removed from the received signal such that neither $\widetilde{a}_{L M M S E}\left(p^{\prime}+i M, q\right)$ nor $\widetilde{a}_{M I C}\left(p^{\prime}+i M, q\right)$ is the linear MMSE estimate of $a\left(p^{\prime}+i M, q\right)$ given the equivalent received signal written as

$$
\begin{aligned}
& \ddot{\mathbf{y}}_{w}\left(p^{\prime}+i M, q\right) \\
& \left.\equiv \mathbf{y}_{w}(i)-\sum_{u=0, u \neq p^{\prime}} \sum_{v=0, v \neq q}^{N_{c}-1} a(u+i M, v) \mathbf{s}_{w}(u, v)\right] \\
& =a\left(p^{\prime}+i M, q\right) \mathbf{s}_{w}\left(p^{\prime}, q\right)+\mathbf{n}_{w}(i)+\mathbf{i}_{w}(i), \\
& \quad p^{\prime}=0,1, \ldots, M-1, \quad q=0,1, \ldots, N_{c}-1
\end{aligned}
$$

where $\mathbf{n}_{w}(i)$ and $\mathbf{i}_{w}(i)$ denote the signal components contributed by AWGN and CCI respectively. Motivated by this observation, we propose the EMIC algorithm which is the 
linear MMSE estimate of the information bit $a\left(p^{\prime}+i M, q\right)$ given the equivalent received signal in (11).

The estimate of the information bit $a\left(p^{\prime}+i M, q\right), p^{\prime}=$ $0,1, \ldots, M-1, q=0,1, \ldots, N_{c}-1$, at the output of the second stage of the EMIC has the form

$$
\begin{aligned}
& \widetilde{a}_{E M I C}\left(p^{\prime}+i M, q\right) \\
& =\mathbf{v}_{E M I C}^{T}\left(p^{\prime}+i M, q\right) \\
& \quad\left[\mathbf{y}_{w}(i)-\sum_{u=0, u \neq p^{\prime}}^{M-1} \sum_{v=0, v \neq q}^{N_{c}-1} \hat{a}(u+i M, v) \mathbf{s}_{w}(u, v)\right]
\end{aligned}
$$

where $\mathbf{v}_{E M I C}^{T}\left(p^{\prime}+i M, q\right)$ is chosen to minimized the MSE given as

$$
\begin{aligned}
& \mathbf{v}_{E M I C}\left(p^{\prime}+i M, q\right) \\
& =\arg \min _{\mathbf{v} \in C^{L M \times 1}} E\left[\left|a\left(p^{\prime}+i M, q\right)-\mathbf{v}^{T} \ddot{\mathbf{y}}_{w}\left(p^{\prime}+i M, q\right)\right|^{2}\right] .
\end{aligned}
$$

Following the same optimization procedure as in (5), we have

$$
\begin{aligned}
& \mathbf{v}_{E M I C}\left(p^{\prime}+i M, q\right) \\
& =\mathbf{s}_{w}^{H}\left(p^{\prime}, q\right) E^{-1}\left[\mathbf{s}_{w}\left(p^{\prime}, q\right) \mathbf{s}_{w}^{H}\left(p^{\prime}, q\right)+\mathbf{n}_{w} \mathbf{n}_{w}^{H}+\mathbf{i}_{w} \mathbf{i}_{w}^{H}\right] \\
& =\mathbf{s}_{w}^{H}\left(p^{\prime}, q\right)\left[\mathbf{R}-\sum_{u=0, u \neq p^{\prime}}^{M-1} \sum_{v=0, v \neq q}^{N_{c}} \mathbf{s}_{w}(u, v) \mathbf{s}_{w}^{H}(u, v)\right]^{-1}
\end{aligned}
$$

Assuming that tentative decisions are perfect and denoting $\ddot{\mathbf{R}}\left(p^{\prime}, q\right) \equiv\left[\mathbf{R}-\sum_{u=0, u \neq p^{\prime}}^{M-1} \sum_{v=0, v \neq q}^{N_{c}} \mathbf{s}_{w}(u, v) \mathbf{s}_{w}^{H}(u, v)\right]$, the output SINR of the EMIC of the information bit $a\left(p^{\prime}+i M, q\right)$, $p^{\prime}=0,1, \ldots, M-1, q=0,1, \ldots, N_{c}-1$, is shown to be

$$
\begin{aligned}
& \operatorname{SINR}_{E M I C}\left(p^{\prime}+i M, q\right) \\
& =\frac{\left[\mathbf{s}_{w}^{H}\left(p^{\prime}, q\right) \ddot{\mathbf{R}}^{-1} \mathbf{s}_{w}\left(p^{\prime}, q\right)\right]^{2}}{\mathbf{s}_{w}^{H}\left(p^{\prime}, q\right) \ddot{\mathbf{R}}^{-1} \mathbf{s}_{w}\left(p^{\prime}, q\right)-\left[\mathbf{s}_{w}^{H}\left(p^{\prime}, q\right) \ddot{\mathbf{R}}^{-1} \mathbf{s}_{w}\left(p^{\prime}, q\right)\right]^{2}} \\
& =\mathbf{s}_{w}^{H}\left(p^{\prime}, q\right) E^{-1}\left[\mathbf{n}_{w} \mathbf{n}_{w}^{H}+\mathbf{i}_{w} \mathbf{i}_{w}^{H}\right] \mathbf{s}_{w}\left(p^{\prime}, q\right)
\end{aligned}
$$

which is independent of other sub-carriers since the signal components from other sub-carriers are completely removed. Although only two stages of the MIC and the EMIC are shown in the derivations, the multistage approaches can be further iterated, by cleaning up the residual interference with, hopefully, increasingly reliable tentative decisions.

\section{NUMERICAL RESUlTS}

In this section, we assess the BER performance of the proposed algorithms by computer simulations. Since we primarily focus on the development of interference cancellation algorithms in this paper, the parameter estimation of the algorithms is temporally not taken care such that $\mathbf{v}_{L M M S E}^{T}\left(p^{\prime}+i M, q\right)$ and $\mathbf{v}_{E M I C}^{T}\left(p^{\prime}+i M, q\right)$ are assumed precisely known to the receiver throughout this section. Although the MIC and the EMIC are multistage algorithms, we only show the simulations of their two-stage versions.

\section{A. Simulation Setup}

We consider OFDM signals operating at ISM band with quadrature phase-shift keying (QPSK) and 16-ary quadrature amplitude modulation (16-QAM) as two numerical examples where $N_{c}=48, T_{0}=4 \mu \mathrm{sec}$ and $T_{1}=0.8 \mu \mathrm{sec}$. The desired signals employ a rate $1 / 2$ binary convolutional code with generators in octal $\left[133_{8}, 171_{8}\right]$. The CCI is an asynchronous DS/CDMA signal with differential quadrature phase-shift keying (DQPSK) modulation from an IEEE $802.11 \mathrm{~b}$ WLAN system and its frequency offset relative to the desired signal is zero. The channel coefficients of the DS/CDMA signal are also assumed time-invariant in our observation interval such that the interference is cyclostationay with period $4 \mu \mathrm{sec}$. We choose the period of the sliding window $M=1$. The OFDM signal and interference are approximated as $20 \mathrm{Mhz}$ so that $L=64 . T_{d}=0.75 \mu \mathrm{sec}$ in order to avoid ISI and ICI. As the channel models exclusively applied in WLAN [17], the tap coefficients are i.i.d complex-valued Gaussian random variables with zero mean and exponentially decayed variance. The channel models of the DS/CDMA signal and the OFDM signal are common and the coefficients are generated independently in the simulations. The receiver employs a harddecision Viterbi decoder of traceback length 128 at each stage.

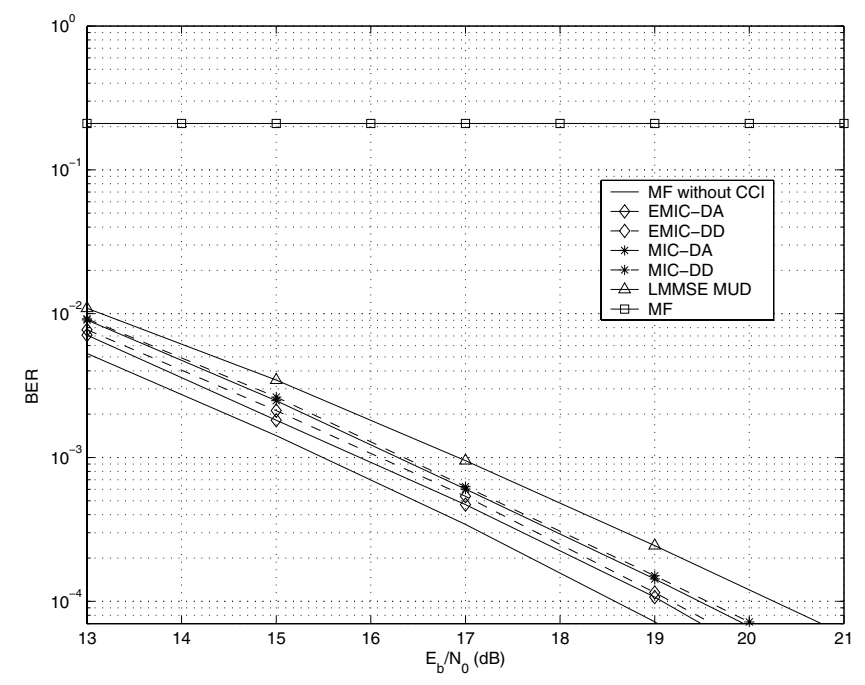

Fig. 1. BER versus $E_{b} / N_{0}$ of the proposed algorithms for the convolutional coded OFDM system with QPSK modulation and code rate $1 / 2$ in the presence of asynchronous $\mathrm{CCI}$ with $\mathrm{SIR}=0 \mathrm{~dB}$.

\section{B. Results}

In addition to the proposed DD algorithms, the BER curves of their corresponding DA versions, namely the MIC-DA and the EMIC-DA, and the matched filter (MF) algorithm are also shown in Figs. 1 and 2 when the signal-to-interference ratio (SIR) is $0 \mathrm{~dB}$. The performance of the MF in the absence of CCI is also shown as the lower bound of the optimal performance. For the case of QPSK shown in Fig. 1, the slope of the BER curve of the LMMSE MUD only slightly decreases compared to the lower bound, which shows the effectiveness 
of the LMMSE MUD. The performance loss of the LMMSE MUD relative the lower bound is $1.8 \mathrm{~dB}$ at the target BER $10^{-4}$. Thanks to the reliable tentative decisions from the first stage, the performance of the MIC-DD and EMIC-DD is quite close to their corresponding DA versions. At the target BER, the performance loss of MIC-DD is $1 \mathrm{~dB}$ relative to the lower bound. The EMIC-DD further reduces the performance loss to $0.5 \mathrm{~dB}$.

For the case of 16-QAM shown in Fig. 2, since the modulation schemes with higher signal constellations are naturally more sensitive to the residual interference, the slope of the BER curve of the LMMSE MUD greatly decreases compared to the lower bound. The performance loss of the LMMSE MUD relative to the lower bound increases to $5 \mathrm{~dB}$ at the BER $10^{-4}$. By cancelling the residual interference at the output of the LMMSE MUD effectively, the performance gain of the MIC-DA and MIC-DD over the LMMSE MUD are 3.2dB and $2.3 \mathrm{~dB}$ respectively. Remarkably, the performance loss of EMIC-DA relative to the lower bound is only $0.5 \mathrm{~dB}$. Although the EMIC-DD seems more sensitive to the error of tentative decisions especially in the lower $E_{b} / N_{0}$ range, it further increases the performance gain over LMMSE MUD to $3.2 \mathrm{~dB}$ at the target BER. And we can expect further performance improvement of EMIC-DD if the number of stages increases.

\section{CONCLUSIONS}

In this paper, we proposed the LMMSE MUD, the MIC and the EMIC algorithms to mitigate severe CCI for coded OFDM systems over single input/single output frequencyselective slowly fading channels. As shown in the simulations, with the proposed algorithms, OFDM systems with QPSK and 16-QAM modulation schemes are resistent to the wide-band asynchronous DS/CDMA signal with DQPSK modulation scheme from an IEEE $802.11 \mathrm{~b}$ WLAN system. Among the proposed algorithms, the EMIC algorithm shows a remarkable performance improvement such that its BER curves are quite close to the lower bounds for both QPSK and 16-QAM cases. In addition to study the implementation complexity of the EMIC, we will investigate the properties of the proposed algorithms against narrow-band CCI in wireless coded OFDM systems and possibly power-line OFDM systems. And finally we will extend the proposed algorithms to coded OFDM systems with antenna arrays.

\section{REFERENCES}

[1] Y. Li, and N. R. Sollenberger, "Adaptive antenna arrays for OFDM systems with co-channel interference," IEEE Trans. Commun, vol. 47, pp. 217-229, Feb. 1999.

[2] S. KAPOOR, D. J. Marchok, and Y. Huang, "Adaptive interference suppression in multiuser wireless OFDM systems using antenna arrays," IEEE Trans. Signal Processing, vol. 47, pp. 3381-3391, Dec. 1999.

[3] J. Cheng, Y. Kamiya, and T. Ohira, "Adaptive beamforming of ESPAR antenna based on steepest gradient algorithm," IEICE Trans. Commun., vol. E84-B, pp. 1790-1800, July 2001.

[4] M. Speth, A. Senst, and H. Meyr, "Low Complexity Space-Frequency MLSE For Multi-User COFDM," IEEE Proc. of Global Telecommunications Conference, vol. 1, pp. 2395-2399, Dec. 1999.

[5] P. Vandenameele, L. V. der Perre, M. Engles, B.Gyselinckx, and H. D. Man, "A Combined OFDM/SDMA Approach," IEEE J. Select. Areas Commun., vol. 18, pp. 2312-2321, Nov. 2000.

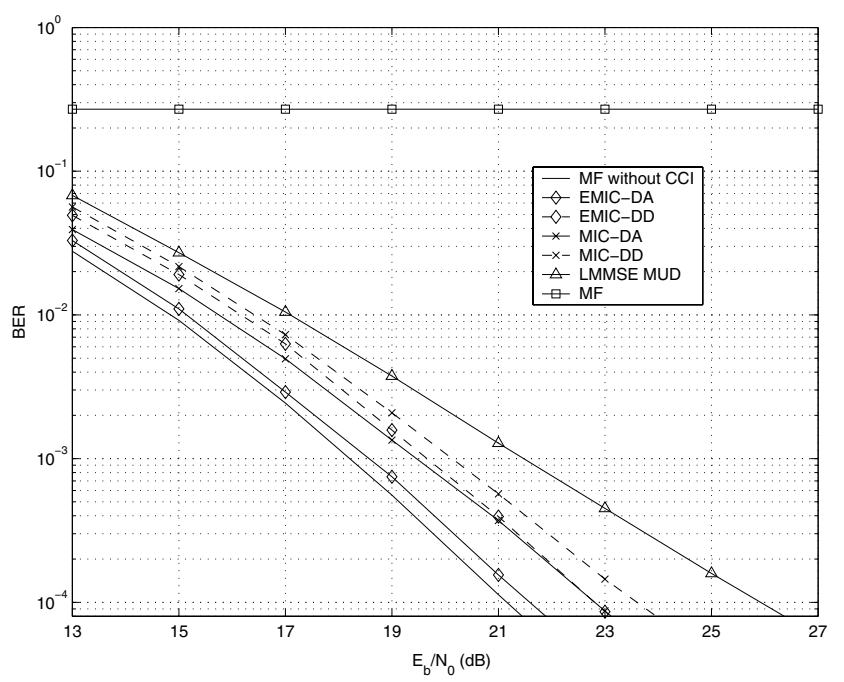

Fig. 2. BER versus $E_{b} / N_{0}$ of the proposed algorithms for the convolutional coded OFDM system with 16 -QAM modulation and code rate $1 / 2$ in the presence of asynchronous $\mathrm{CCI}$ with $\mathrm{SIR}=0 \mathrm{~dB}$.

[6] M. Münster and L. Hanzo, "Co-channel interference cancellation techniques for antenna array assisted multiuser OFDM systems," Proc. of $3 G$ Mobile Communication Technologies Conference, pp. 256-260, Mar. 2000.

[7] S. Verdu, Multiuser Detection, Cambridge, U.K.: Cambridge Univ. Press, 1998.

[8] M. Honig, U. Madhow, and S. Verdu, "Blind multiuser detection," IEEE Trans. Inform. Theory, vol. IT-41, pp. 944-960, July 1995.

[9] V. Poor and X. Wang, "Code-aided interfernce suppression for DS/CDMA communications-Part I: Interference suppression capability," IEEE Trans. Commun., vol. 45, pp. 1101-1111, Sept. 1997.

[10] V. Poor and X. Wang, "Code-aided interfernce suppression for DS/CDMA communications-Part II: Parallel blind adaptive implementations," IEEE Trans. Commun., vol. 45, pp. 1112-1122, Sept. 1997.

[11] S. Buzzi, M. Lops, and A. M. Tulino, "Time-varying narrow-band interference rejection in asynchronous multiuser DS/CDMA systems over frequency selective fading channels," IEEE Trans. Commun., vol. 47, pp. 1523-1536, Oct. 1999.

[12] J. G. Proakis, Digital Communications, 2nd ed., New York: McGrawHill, 1989.

[13] U. Madhow and M. L. Honig, "MMSE interference suppression for direct-sequence spread spectrum CDMA," IEEE Trans. Commun., vol. 42, pp. 3178-3188, Dec. 1994.

[14] S. Haykin, Adaptive Filtering Theory, 3rd ed. Englewood Cliffs, NJ: Prentice-Hall, 1996.

[15] G. Caire, "Two-stage nondata-aided adaptive linear reveivers for DS/CDMA," IEEE Trans. Cummun., vol. 48, pp. 1712-1724, Oct. 2000.

[16] X. Zhang and W. Wei, "Blind adaptive multiuser detectin based on Kalman filtering," IEEE Trans. Signal Processing, vol. 50, pp-87-95, Jan. 2002

[17] B. O'Hara and A. Petrick, "The IEEE 802.11 handbook: A designer's companion," Standard Information Network IEEE Press, 1999.

[18] Supplement to IEEE Standard for Information technology - Part 11:Wireless LAN Medium Access Control (MAC) and Phisical Layer (PHY) specifications: Further Higher-Speed Physical Layer Extension in the $2.4 \mathrm{GHz}$ Band. 